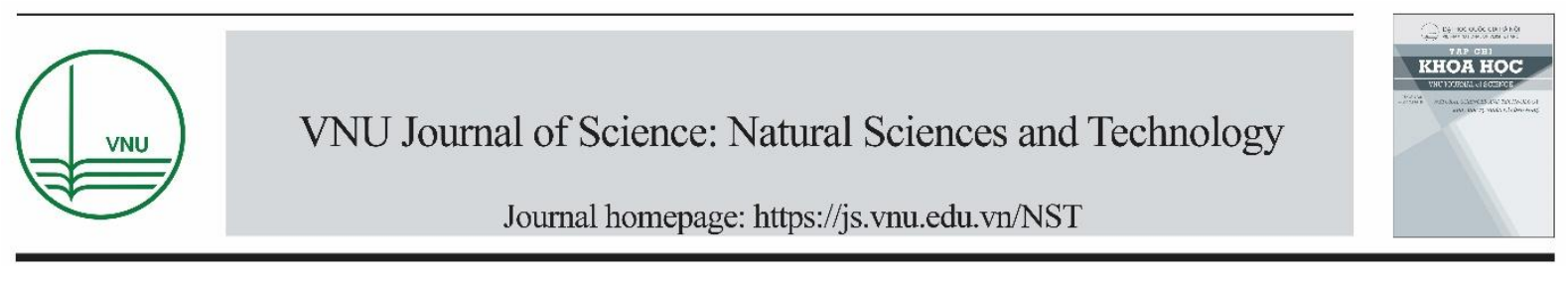

Original Article

\title{
Triterpenoids and Phytosterols Isolated from Pluchea Indica L. Leaves
}

\author{
Do Thi Viet Huong ${ }^{1}$, Phan Minh Giang ${ }^{1, *}$, Hoang Thi Sim ${ }^{1}$, Truong Thi To Chinh ${ }^{2}$ \\ ${ }^{1}$ Faculty of Chemistry, VNU University of Science, 19 Le Thanh Tong, Hanoi, Vietnam \\ ${ }^{2}$ Vietnam Institute of Industrial Chemistry, 2 Pham Ngu Lao, Phan Chu Trinh, Hanoi, Vietnam
}

Received 25 May 2019

Revised 12 June 2019; Accepted 13 June 2019

\begin{abstract}
Taraxasterol acetate, 1-dotriacontanol, taraxasterol, stigmasterol, 2-(prop-1-ynyl)-5-(5,6dihyroxyhexa-1,3-diynyl)thiophene), stigmasterol 3-O- $\beta$-D-glucopyranoside, and $\beta$-sitosterol 3-O$\beta$-D-glucopyranoside were isolated from the leaves of Pluchea indica and determined with NMR techniques. Taraxasterol acetate, taraxasterol, and stigmasterol could be isolated in high yields from Pluchea indica growing in Gia Lam, Hanoi, Vietnam.
\end{abstract}

Keywords: Pluchea indica, phytosterol, triterpenoid, taraxasterol, stigmasterol.

${ }^{*}$ Corresponding author.

Email address: phanminhgiang@yahoo.com

https://doi.org/10.25073/2588-1140/vnunst.4910 


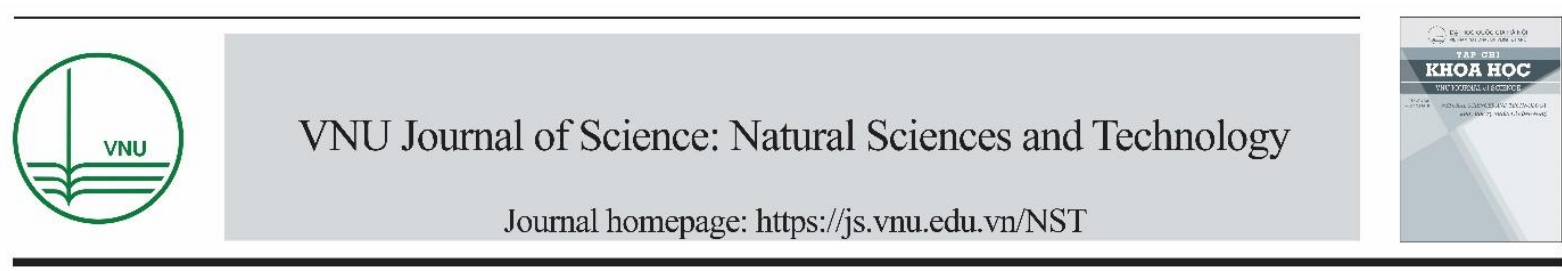

\title{
Triterpenoid và phytosterol từ lá cây Cúc Tần (Pluchea indica L.)
}

\author{
Đỗ Thị Việt Hương ${ }^{1}$,Phan Minh Giang ${ }^{1, *}$, Hoàng Thị Sim¹, Trương Thị Tố Chinh² \\ ${ }^{1}$ Khoa Hóa học, Truờng Đại học Khoa học Tụ nhiên, Đại học Quốc gia Hà Nội, \\ 19 Lê Thánh Tông, Hoàn Kiếm, Hà Nội, Việt Nam \\ ${ }^{2}$ Viện Hóa học Công nghiệp Việt Nam, 2 Phạm Ngũ Lão, Phan Chu Trinh, Hoàn Kiếm, Hà Nội, Việt Nam
}

Nhận ngày 25 tháng 5 năm 2019

Chỉnh sửa ngày 12 tháng 6 năm 2019; Chấp nhận đăng ngày 13 tháng 6 năm 2019

\begin{abstract}
Tóm tắt: Taraxasteryl acetate, 1-dotriacontanol, taraxasterol, stigmasterol, 2-(prop-1-ynyl)-5-(5,6dihyroxyhexa-1,3-diynyl)thiophene), stigmasterol 3-O- $\beta$-D-glucopyranoside và $\beta$-sitosterol 3-O- $\beta$ D-glucopyranoside được phân lập từ lá của cây cúc tần (Pluchea indica $\mathrm{L}$.), các hợp chất này được nhận dạng dựa vào phương pháp cộng hưởng từ hạt nhân NMR. Hàm lượng các hợp chất taraxasteryl acetate, taraxasterol, và stigmasterol phân lập được trong lá cây cúc tần được thu hái ở Gia Lâm, Hà Nôi tương đối cao.
\end{abstract}

Tù khóa: Pluchea indica, phytosterol, triterpenoid, taraxasteran, stigmastane.

\section{Mở đầu}

Chi Pluchea thuộc họ Cúc (Asteraceae) có khoảng 10 loài ở châu Á. Ở Việt Nam, chi Pluchea có 4 loài phổ biến: P. eupatorioides Kurz. (cây lức, nát cam), $P$. polygonata Gagnep. (cây cúc bông, hoa mật), P. pteropoda Hemsl. (cây sài hồ nam, nam sài hồ), $P$. indica $\mathrm{L}$. (cây cúc tần, từ bi) [1]. Nghiên cứu thành phần hóa học chi Pluchea xác định được các thành phần sesquiterpenoid, flavonoid, acid caffeoylquinic, steroid, triterpenoid, diterpenoid, và alkynyl thiophen [2]. Nghiên cứu cây $P$. indica xác định được nhiều hợp chất mới với hoạt tính kháng viêm, kháng khuẩn, chống ung thư, ức chế enzym acetylcholinesterase [2]. Từ cây Cúc tần Việt Nam tinh dầu [3] và 2-(prop-1-ynyl)-5-(5,6dihydroxyhexa-1,3-diynyl)nthiophen [4] đã được phân lập. Nhằm góp phần nghiên cứu đầy đủ hơn về thành phần hóa học của cây $P$. indica ở Việt Nam chúng tôi đã thu thập mẫu lá cây Cúc tần miền Bắc và thực hiện việc phân lập các hợp chất terpenoid và phytosterol. Kết quả là đã phân lập được các hợp chất thành phần terpenoid chính nhóm taraxasteran và phytosterol chính nhóm stigmastan (Hình 1).

\footnotetext{
*Tác giả liên hệ.

Địa chỉ email: phanminhgiang@yahoo.com
}

https://doi.org/10.25073/2588-1140/vnunst.4910 

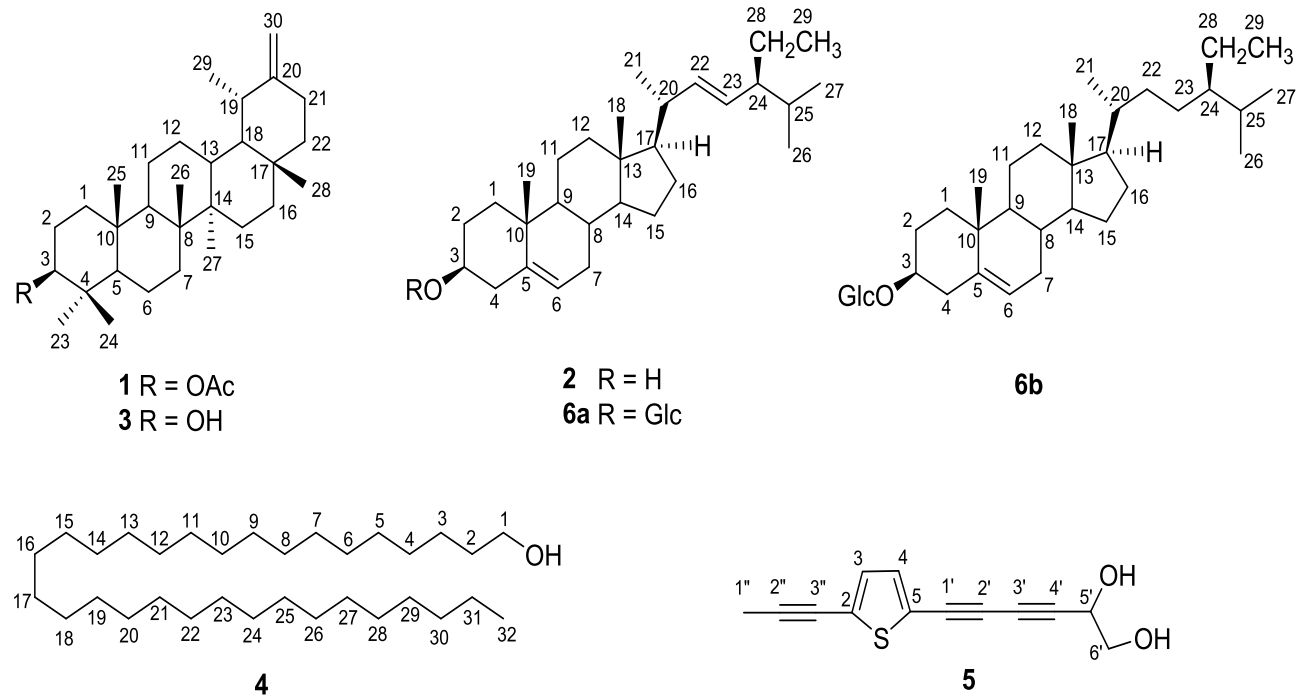

Hình 1. Cấu trúc các hợp chất 1-6.

\section{Thiết bị và phương pháp}

\subsection{Thiết bị và hóa chất}

Phổ khối lượng phun bụi điện (ESI-MS) được ghi trên hệ thống LC/MSD Trap Agilen Series 1100. Phổ cộng hưởng từ hạt nhân proton (1H-NMR, $500 \mathrm{MHz}$ ), carbon-13 (13C-NMR, $125 \mathrm{MHz})$, DEPT được ghi trên thiết bị Bruker Avance 500 với tetramethylsilan (TMS) là chất chuẩn nội. Độ chuyển dịch hoá học $\delta$ được biểu thị bằng $\mathrm{ppm}, \mathrm{J}$ tính theo $\mathrm{Hz}$. Sắc ký lớp mỏng (TLC) được thực hiện trên bản mỏng tráng sẵn DC-Alufolien 60 F254 (Merck, Darmstadt, Đức). Phát hiện vệt chất bằng thuốc thử vanilin/H2SO4 đặc 1\%. Sắc ký cột $(\mathrm{CC})$ được thực hiện trên chất hấp phụ silica gel (Merck, Darmstadt, Đức) với các cỡ hạt 63-200, 40-63 và $15-40 \mu \mathrm{m}$.

\subsection{Nguyên liệu nghiên cứu}

Mẫu cây Cúc tần ( $P$. indica $\mathrm{L}$.) được thu hái vào tháng 9 năm 2015 tại Gia Lâm, Hà Nội. Mẫu được TS. Nguyễn Thị Kim Thanh, Khoa Sinh học, Trường Đại học Khoa học Tự nhiên, Đại học Quốc gia Hà Nội giám định thực vật. Lá sau đó được tách bỏ cành, phơi trong bóng râm rồi sấy ở nhiệt độ $45^{\circ} \mathrm{C}$ đến khô và được xay thành bột.

\section{3. Điều chế các phần chiết}

Ngâm chiết bột lá khô cây Cúc tần $(2,5 \mathrm{~kg})$ trong methanol ở nhiệt độ phòng trong 3 ngày, quy trình được lặp lại 3 lần. Gộp các dịch lọc rồi cất loại dung môi dưới áp suất giảm cho cặn chiết methanol. Cặn chiết này được hòa với nước rồi chiết lần lượt với các dung môi $n$-hexan và dichloromethan theo độ phân cực tăng dần cho các dịch chiết hữu cơ tương ứng. Cất loại các dịch chiết dưới áp suất giảm cho các phần chiết $n$-hexan $(96,1 \mathrm{~g})$ và dichloromethan $(19,6 \mathrm{~g})$. Phần chiết $n$-hexan $(96,1 \mathrm{~g})$ được phân tách sắc ký CC với hệ dung môi gradient $n$-hexan-aceton 49:1, 29:1, 19:1, 9:1, 4:1, 2:1, 1:1 cho 9 nhóm phân đoạn FH1-FH9. Các nhóm phân đoạn FH2 và $\mathrm{FH} 4$ được rửa nhiều lần bằng $n$-hexan cho tương ứng 1 (600 $\mathrm{mg})$ và 2 (725 mg). Nhóm phân đoạn $\mathrm{FH} 3$ được phân tách sắc ký $\mathrm{CC}$ với hệ dung môi gradient $n$-hexan-EtOAc 29:1, 19:1, 9:1, 6:1, 4:1, 2:1 cho 8 nhóm phân đoạn FH31-FH38. Nhóm phân đoạn $\mathrm{FH} 34$ được rửa bằng aceton cho 3 (465 mg). Nhóm phân đoạn FH36 được rửa bằng $n$-hexan cho $2(25 \mathrm{mg})$. Hai nhóm phân đoạn $\mathrm{FH} 32$ và $\mathrm{FH} 33$ được gộp lại và phân tách sắc ký $\mathrm{CC}$ với hệ dung môi gradient $n$-hexanEtOAc 49:1, 29:1, 19:1 cho 4 nhóm phân đoạn FH321-FH324. Nhóm phân đoạn FH323 được 
phân tách sắc kí $\mathrm{CC}$, rửa giải bằng $n$-hexanEtOAc 49:1 cho 4 (30 mg). Nhóm phân đoạn FH8 được phân tách sắc ký CC với hệ dung môi gradient $\mathrm{CH}_{2} \mathrm{Cl}_{2}$-EtOAc 90:1, 70:1, 49:1, 29:1, 19:1, 9:1, 4:1, 2:1 cho 8 nhóm phân đoạn FH81FH88. Nhóm phân đoạn FH85 được phân tách sắc ký $\mathrm{CC}$ với hệ dung môi gradient $\mathrm{CH}_{2} \mathrm{Cl}_{2}-$ aceton 49:1, 29:1, 19:1, 9:1, 4:1 cho 4 nhóm phân đoạn FH851-854. Nhóm phân đoạn FH853 được phân tách sắc ký $\mathrm{CC}$ với hệ dung môi gradient $\mathrm{CH}_{2} \mathrm{Cl}_{2}$-aceton 70:1, 49:1, 29:1, 19:1, 9:1, 4:1 cho 2 nhóm phân đoạn F8531 và F8532. Nhóm phân đoạn FH8532 được tinh chế CC với hệ dung môi gradient $n$-hexan-EtOAc 9:1, 6:1, 4:1, 2:1, 1:1 cho 5 (10 mg). Nhóm phân đoạn FH87 được phân tách sắc ký $\mathrm{CC}$ với hệ dung môi gradient $\mathrm{CH}_{2} \mathrm{Cl}_{2}$-aceton 9:1, 6:1, 4:1, 3:1, 2:1 cho 4 nhóm phân đoạn FH871-FH874. Nhóm phân đoạn FH873 được rửa bằng $n$-hexan cho 6 (15 mg). Nhóm phân đoạn FH88 được phân tách sắc ký $\mathrm{CC}$ với hệ dung môi gradient $\mathrm{CH}_{2} \mathrm{Cl}_{2}$ aceton $6: 1,4: 1,3: 1,2: 1$ cho 4 nhóm phân đoạn FH881-FH884. Nhóm phân đoạn FH884 được tinh chế bằng $\mathrm{CC}$ với hệ dung môi gradient $\mathrm{CH}_{2} \mathrm{Cl}_{2}$-aceton 3:1, 2:1, 1:1 cho 6 (15 mg). Phần chiết dichloromethan $(19,6 \mathrm{~g})$ được phân tách $\mathrm{CC}$ với hệ dung môi gradient $\mathrm{CH}_{2} \mathrm{Cl}_{2}$-aceton 29:1, 19:1, 9:1, 6:1, 4:1, 2:1 cho 3 nhóm phân đoạn FD1-FD3. Nhóm phân đoạn FD2 được phân tách CC với hệ dung môi gradient $n$-hexanEtOAc 29:1, 19:1, 9:1, 4:1, 2:1 cho 9 nhóm phân đoạn FD21-FD29. Nhóm phân đoạn FD21 được tinh chế CC với hệ dung môi gradient $n$-hexanEtOAc 9:1, 6:1, 4:1, 2:1, 1:1 cho 1 (10 mg). Nhóm phân đoạn FD23 được rửa bằng $n$-hexan cho $4(5 \mathrm{mg})$. Nhóm phân đoạn FD25 được rửa bằng $n$-hexan cho $2(6 \mathrm{mg})$.

Taraxasteryl acetat (1): Tinh thể hình lá trắng bạc, t.n.c. $248-249{ }^{\circ} \mathrm{C} . \mathrm{R}_{f}=0,55$ (TLC, silica gel, $n$-hexan-aceton 49:1, v/v). ${ }^{1} \mathrm{H}-\mathrm{NMR}$ $\left(\mathrm{CDCl}_{3}\right): \delta$ (ppm) $0,84\left(3 \mathrm{H}, \mathrm{s}, \mathrm{CH}_{3}-23\right), 0,85$ $\left(3 \mathrm{H}, \mathrm{s}, \mathrm{CH}_{3}-24\right), 0,86\left(3 \mathrm{H}, \mathrm{s}, \mathrm{CH}_{3}-28\right), 0,88(3 \mathrm{H}$, $\left.\mathrm{s}, \mathrm{CH}_{3}-25\right), 0,93\left(3 \mathrm{H}, \mathrm{s}, \mathrm{CH}_{3}-27\right), 1,02(3 \mathrm{H}, \mathrm{s}$, $\left.\mathrm{CH}_{3}-26\right), 1,03$ (3H, d, $\left.J=7,0 \mathrm{~Hz}, \mathrm{CH}_{3}-29\right), 2,04$ $(3 \mathrm{H}, \mathrm{s}, 3-\mathrm{OAc}), 4,49(1 \mathrm{H}, \mathrm{dd}, J=6,3 \mathrm{~Hz}, 10,0$ $\mathrm{Hz}, \mathrm{H}-3), 4,60$ (1H, s br, H-30a), 4,62 (1H, s br, $\mathrm{H}-30 \mathrm{~b}) .{ }^{13} \mathrm{C}-\mathrm{NMR}\left(\mathrm{CDCl}_{3}\right): \delta$ (ppm) $14,8(\mathrm{C}-$
27), 15,9 (C-25), 16,4 (C-26), 16,5 (C-24), 18,2 (C-6), 19,5 (C-29), 21,3 (3-OAc), 21,5 (C-11), 23,7 (C-2), 25,5 (C-21), 25,7 (C-12), 26,2 (C28), 26,7 (C-15), 27,9 (C-23), 34,0 (C-7), 34,6 (C-17), 37,1 (C-10), 37,8 (C-4), 38,3 (C-19), 38,5 (C-1), 38,9 (C-13), 39, 2 (C-16), 39,4 (C22), 40,9 (C-8), 42,0 (C-14), 48,7 (C-18), 50,8 (C-9), 55,5 (C-5), 81,1 (C-3), 107,1 (C-30), 154,7 (C-20), 171,1 (3-OAc).

Stigmasterol (2): Tinh thể hình kim màu trắng, t.n.c. $160-162{ }^{\circ} \mathrm{C} \cdot \mathrm{R}_{f}=0,41(\mathrm{TLC}$, silica gel, $n$-hexan-aceton $4: 1$, v/v). ${ }^{1} \mathrm{H}-\mathrm{NMR}\left(\mathrm{CDCl}_{3}\right)$ : $\delta(\mathrm{ppm}) 0,69\left(3 \mathrm{H}, \mathrm{s}, \mathrm{CH}_{3}-19\right), 0,79(3 \mathrm{H}, \mathrm{d}, J=$ $\left.7,0 \mathrm{~Hz}, \mathrm{CH}_{3}-26\right), 0,81\left(3 \mathrm{H}, \mathrm{t}, J=7,0 \mathrm{~Hz}, \mathrm{CH}_{3}-\right.$ 29), 0,85 (3H, d, $\left.J=7,0 \mathrm{~Hz}, \mathrm{CH}_{3}-27\right), 1,01(3 \mathrm{H}$, $\left.\mathrm{s}, \mathrm{CH}_{3}-18\right), 1,02\left(3 \mathrm{H}, \mathrm{d}, J=6,5 \mathrm{~Hz}, \mathrm{CH}_{3}-21\right)$, $3,53(1 \mathrm{H}, \mathrm{tt}, J=4,0 \mathrm{~Hz}, 11,0 \mathrm{~Hz}, \mathrm{H}-3), 5,02(1 \mathrm{H}$, dd, $J=8,5 \mathrm{~Hz}, 15,0 \mathrm{~Hz}, \mathrm{H}-23), 5,15(1 \mathrm{H}, \mathrm{dd}, J=$ $8,5 \mathrm{~Hz}, 15,0 \mathrm{~Hz}, \mathrm{H}-22), 5,35(1 \mathrm{H}, \mathrm{d}$ br, $J=5,0$ $\mathrm{Hz}, \mathrm{H}-6)$.

Taraxasterol (3): Tinh thể hình que màu trắng, t.n.c. $220-221{ }^{\circ} \mathrm{C} \cdot \mathrm{R}_{f}=0,41$ (TLC, silica gel, $n$-hexan-EtOAc $9: 1, \quad$ v/v). ${ }^{1} \mathrm{H}-\mathrm{NMR}$ $\left(\mathrm{CDCl}_{3}\right): \delta(\mathrm{ppm}) \quad 0,77\left(3 \mathrm{H}, \mathrm{s}, \mathrm{CH}_{3}-24\right), 0,85$ $\left(3 \mathrm{H}, \mathrm{s}, \mathrm{CH}_{3}-28\right), 0,86\left(3 \mathrm{H}, \mathrm{s}, \mathrm{CH}_{3}-25\right), 0,93(3 \mathrm{H}$, s, $\left.\mathrm{CH}_{3}-27\right), 0,97\left(3 \mathrm{H}, \mathrm{s}, \mathrm{CH}_{3}-23\right), 1,02(3 \mathrm{H}, \mathrm{s}$, $\left.\mathrm{CH}_{3}-26\right), 1,03\left(3 \mathrm{H}, \mathrm{d}, J=7,0 \mathrm{~Hz}, \mathrm{CH}_{3}-29\right), 3,20$ $(1 \mathrm{H}, \mathrm{dd}, J=5,0 \mathrm{~Hz}, 11,0 \mathrm{~Hz}, \mathrm{H}-3), 4,60(1 \mathrm{H}, \mathrm{d}$, $J=2,0 \mathrm{~Hz}, \mathrm{H}-30 \mathrm{a}), 4,61(1 \mathrm{H}, \mathrm{d}, J=2,0 \mathrm{~Hz}$, $\mathrm{H}-30 \mathrm{~b})$.

1-Dotriacontanol (4): Bột vô định hình màu trắng. $\mathrm{R}_{f}=0,3$ (TLC, silica gel, $n$-hexan-EtOAc 19:1, v/v). ${ }^{1} \mathrm{H}-\mathrm{NMR}\left(\mathrm{CDCl}_{3}\right): \delta(\mathrm{ppm}) 0,87(3 \mathrm{H}$, $\left.\mathrm{t}, J=6,5 \mathrm{~Hz}, \mathrm{CH}_{3}-32\right), 1,25\left(58 \mathrm{H}, \mathrm{s} \mathrm{br}, \mathrm{CH}_{2}-3-\right.$ $\mathrm{CH}_{2}-31,1,56\left(2 \mathrm{H}\right.$, quintet, $\left.J=7,5 \mathrm{~Hz}, \mathrm{CH}_{2}-2\right)$, 3,64 (2H, t, $\left.J=6,5 \mathrm{~Hz}, \mathrm{CH}_{2}-1\right)$. ESI-MS: $\mathrm{m} / z$ $489,3[\mathrm{M}+\mathrm{Na}]^{+}\left(\mathrm{C}_{32} \mathrm{H}_{66} \mathrm{O}\right)$.

2-(Prop-1-ynyl)-5-(5,6-dihyroxyhexa-1,3diynyl)-thiophen (5): Bột vô định hình màu trắng. $\mathrm{R}_{f}=0,4$ (TLC, silica gel, $\mathrm{CH}_{2} \mathrm{Cl}_{2}$-EtOAc $3: 1, \mathrm{v} / \mathrm{v}) .{ }^{1} \mathrm{H}-\mathrm{NMR}\left(\mathrm{CDCl}_{3}\right): \delta(\mathrm{ppm}) 2,04(3 \mathrm{H}$, s, H-1"), 2,25 (1H, s br, -OH), 2,60 (1H, s br, $\mathrm{OH}), 3,77$ (1H, m, H-6'a), 3,81 (1H, m, H-6'b), 4,68 (1H, s, H-5'), 7,04 (1H, d, $J=4,0 \mathrm{~Hz}, \mathrm{H}-3)$, 7,10 $(1 \mathrm{H}, \mathrm{d}, J=4,0 \mathrm{~Hz}, \mathrm{H}-4)$. ESI-MS: $m / z$ $253,22[\mathrm{M}+\mathrm{Na}]^{+}\left(\mathrm{C}_{13} \mathrm{H}_{10} \mathrm{O}_{2} \mathrm{SNa}\right)$. 
Stigmasterol 3-O- $\beta$-D-glucopyranosid $+\beta$ Sitosterol 3-O- $\beta$-D-glucopyranosid (6): Bột vô định hình, màu trắng. $\mathrm{R}_{f}=0,47$ (TLC, silica gel, $\mathrm{CH}_{2} \mathrm{Cl}_{2}$-aceton $\left.3: 1, \mathrm{v} / \mathrm{v}\right)$. ${ }^{1} \mathrm{H}-\mathrm{NMR}\left(\mathrm{CDCl}_{3}+\right.$ $\left.\mathrm{CD}_{3} \mathrm{OD}\right)$ : Stigmasterol 3-O- $\beta$-D-glucopyranosid $(\boldsymbol{6} \boldsymbol{a}): \delta(\mathrm{ppm}) 0,69\left(3 \mathrm{H}, \mathrm{s}, \mathrm{CH}_{3}-18\right), 0,81(3 \mathrm{H}, \mathrm{d}$, $\left.J=7,0 \mathrm{~Hz}, \mathrm{CH}_{3}-26\right), 0,83(3 \mathrm{H}, \mathrm{d}, J=7,0 \mathrm{~Hz}$, $\left.\mathrm{CH}_{3}-27\right), 0,85\left(3 \mathrm{H}, \mathrm{t}, J=7,5 \mathrm{~Hz}, \mathrm{CH}_{3}-29\right), 0,92$ $\left(3 \mathrm{H}, \mathrm{d}, J=6,5 \mathrm{~Hz}, \mathrm{CH}_{3}-21\right), 1,01\left(3 \mathrm{H}, \mathrm{s}, \mathrm{CH}_{3}-\right.$ 19), 3,24 - 3,78 (6H, H-2', H-3', H-4', H-5', 2H$\left.6^{\prime}\right), 3,83(1 \mathrm{H}, \mathrm{dd}, J=3,0 \mathrm{~Hz}, 11,0 \mathrm{~Hz}, \mathrm{H}-3), 4,40$ $\left(1 \mathrm{H}, \mathrm{d}, J=8,0 \mathrm{~Hz}, \mathrm{H}-1^{\prime}\right), 5,37(1 \mathrm{H}, \mathrm{d}$ br, $J=5,0$ $\mathrm{Hz}, \mathrm{H}-6) . \beta$-Sitosterol 3-O- $\beta$-D-glucopyranosid $(\boldsymbol{\sigma} \boldsymbol{b}): \delta(\mathrm{ppm}) 0,70\left(3 \mathrm{H}, \mathrm{s}, \mathrm{CH}_{3}-18\right), 0,79(3 \mathrm{H}, \mathrm{d}$, $\left.J=7,0 \mathrm{~Hz}, \mathrm{CH}_{3}-26\right), 0,83(3 \mathrm{H}, \mathrm{d}, J=7,0 \mathrm{~Hz}$, $\left.\mathrm{CH}_{3}-27\right), 0,84\left(3 \mathrm{H}, \mathrm{t}, J=7,5 \mathrm{~Hz}, \mathrm{CH}_{3}-29\right), 0,92$ $\left(3 \mathrm{H}, \mathrm{d}, J=6,5 \mathrm{~Hz}, \mathrm{CH}_{3}-21\right), 1,01\left(3 \mathrm{H}, \mathrm{s}, \mathrm{CH}_{3}-\right.$ 19), 3,24 - 3,78 (6H, H-2', H-3', H-4', H-5', 2H$\left.6^{\prime}\right), 3,83(1 \mathrm{H}, \mathrm{dd}, J=3,0 \mathrm{~Hz}, 11,0 \mathrm{~Hz}, \mathrm{H}-3), 4,40$ $\left(1 \mathrm{H}, \mathrm{d}, J=8,0 \mathrm{~Hz}, \mathrm{H}-1^{\prime}\right), 5,01(1 \mathrm{H}, \mathrm{dd}, J=8,5$ $\mathrm{Hz}, 15,0 \mathrm{~Hz}, \mathrm{H}-23), 5,15(1 \mathrm{H}, \mathrm{dd}, J=8,5 \mathrm{~Hz}$, $15,0 \mathrm{~Hz}, \mathrm{H}-22), 5,37$ (1H, d br, $J=5,0 \mathrm{~Hz}, \mathrm{H}-6)$.

\section{Kết quả và thảo luận}

Hợp chất 1 đã được phân lập từ phần chiết $n$ hexan dưới dạng tinh thể hình lá trẳng bạc. Phổ ${ }^{1} \mathrm{H}-\mathrm{NMR}\left(\mathrm{CDCl}_{3}\right)$ của $\mathbf{1}$ cho thấy sự có mặt của 6 nhóm methyl bậc 3 ở $\delta_{\mathrm{H}} 0,84(3 \mathrm{H}, \mathrm{s}), 0,85$ (3H, s), 0,86 (3H, s), 0,88 (3H, s), 0,97 (3H, s), 1,02 $(3 \mathrm{H}, \mathrm{s}) ; 1$ nhóm methyl bậc 2 ở $\delta_{\mathrm{H}} 1,021(3 \mathrm{H}, \mathrm{d}$, $J=6,6 \mathrm{~Hz}) ; 1$ nhóm oxymethin ở $\delta_{\mathrm{H}} 4,49(1 \mathrm{H}$, $\mathrm{dd}, J=6,3 \mathrm{~Hz}, 10,0 \mathrm{~Hz}) ; 2$ proton olefinic của một nối đôi ngoại vòng ở $\delta_{\mathrm{H}} 4,60(1 \mathrm{H}, \mathrm{s}), 4,62$ $(1 \mathrm{H}, \mathrm{s}) ; 3$ proton của nhóm acetat ở $\delta_{\mathrm{H}} 2,04(3 \mathrm{H}$, $\mathrm{s})$. So sánh dữ liệu phổ ${ }^{1} \mathrm{H}$ - và ${ }^{13} \mathrm{C}-\mathrm{NMR}$ với tài liệu tham khảo $[5,6], 1$ được xác định là taraxasteryl acetat.

Hợp chất 2 đã được phân lập từ phần chiết $n$ hexan dưới dạng tinh thể hình kim màu trắng. Phổ ${ }^{1} \mathrm{H}-\mathrm{NMR}\left(\mathrm{CDCl}_{3}\right)$ của $\mathbf{2}$ cho thấy sự có mặt của 2 nhóm methyl bậc 3 ở $\delta_{\mathrm{H}} 0,69(3 \mathrm{H}, \mathrm{s}), 1,01$ (3H, s); 1 nhóm methyl bậc 2 ở $\delta_{\mathrm{H}} 1,02$ (3H, d, $J$ $=6,5 \mathrm{~Hz}) ; 2$ nhóm isopropyl ở $\delta_{\mathrm{H}} 0,79(3 \mathrm{H}, \mathrm{d}, J$ $=7,0 \mathrm{~Hz})$ và $0,85(3 \mathrm{H}, \mathrm{d}, J=7,0 \mathrm{~Hz}) ; 1$ nhóm methyl bậc 1 ở $\delta_{\mathrm{H}} 0,81(3 \mathrm{H}, \mathrm{t}, J=7,0 \mathrm{~Hz}) ; 1$ nhóm hydroxymethin ở $\delta_{\mathrm{H}} 3,53(1 \mathrm{H}, \mathrm{tt}, J=4,0$ $\mathrm{Hz}, 11,0 \mathrm{~Hz}) ; 3$ proton olefinic của nối đôi ở $\delta_{\mathrm{H}}$ $5,02(1 \mathrm{H}, \mathrm{d}, J=8,5 \mathrm{~Hz}, 15,0 \mathrm{~Hz}), 5,15(1 \mathrm{H}, \mathrm{d}, J$ $=8,5 \mathrm{~Hz}, 15,0 \mathrm{~Hz})$ và $5,35(1 \mathrm{H}, \mathrm{d} \mathrm{br}, J=5,0 \mathrm{~Hz})$. So sánh phổ ${ }^{1} \mathrm{H}-\mathrm{NMR}$ với tài liệu tham khảo [7], 2 được xác định là stigmasterol.

Hợp chất 3 đã được phân lập từ phần chiết $n$ hexan dưới dạng tinh thể hình que màu trắng. Phổ ${ }^{1} \mathrm{H}-\mathrm{NMR}\left(\mathrm{CDCl}_{3}\right)$ của 3 cho thấy sự có mặt của 6 nhóm methyl bậc 3 ở $\delta_{\mathrm{H}} 0,77(3 \mathrm{H}, \mathrm{s}), 0,85$ $(3 \mathrm{H}, \mathrm{s}), 0,86(3 \mathrm{H}, \mathrm{s}), 0,93(3 \mathrm{H}, \mathrm{s}), 0,97(3 \mathrm{H}, \mathrm{s})$, $1,02(3 \mathrm{H}, \mathrm{s}) ; 1$ nhóm methyl bậc 2 ở $\delta_{\mathrm{H}} 1,08(3 \mathrm{H}$, d, $J=7,1 \mathrm{~Hz}) ; 1$ nhóm hydroxymethin ở $\delta_{\mathrm{H}} 3,2$ $(1 \mathrm{H}, \mathrm{dd}, J=5,0 \mathrm{~Hz}, 11,0 \mathrm{~Hz}) ; 2$ proton olefinic của một nối đôi ngoại vòng ở $\delta_{\mathrm{H}} 4,60(1 \mathrm{H}, \mathrm{d}, J=$ 2,0 Hz), 4,61 (1H, d, $J=2,0 \mathrm{~Hz})$. So sánh phổ ${ }^{1} \mathrm{H}-\mathrm{NMR}$ với tài liệu tham khảo [6], 3 được xác định là taraxasterol.

Hợp chất 4 đã được phân lập từ phần chiết $n$ hexan dưới dạng bột vô định hình màu trắng. Phổ ${ }^{1} \mathrm{H}-\mathrm{NMR}\left(\mathrm{CDCl}_{3}\right)$ của $\mathbf{4}$ cho thấy sự có mặt của 1 nhóm methyl cuối mạch ở $\delta_{\mathrm{H}} 0,87(3 \mathrm{H}, \mathrm{t}, J=$ $6,5 \mathrm{~Hz}) ; 1$ mạch dài hydrocarbon ở $\delta_{\mathrm{H}} 1,25(58 \mathrm{H}$, $\mathrm{s}$ br) và $1,56(2 \mathrm{H}$, quintet, $J=7,5 \mathrm{~Hz}) ; 1$ nhóm hydroxymethylen ở $\delta_{\mathrm{H}} 3,64(2 \mathrm{H}, \mathrm{t}, J=6,5 \mathrm{~Hz}$. Phổ ESI-MS của 4 cho peak ion giả phân tử ở $m / z$ 489,3 $[\mathrm{M}+\mathrm{Na}]^{+}$xác định công thức phân tử $\mathrm{C}_{33} \mathrm{H}_{66} \mathrm{O}$ của alcohol này trong sự phù hợp với phổ ${ }^{1} \mathrm{H}-\mathrm{NMR}$. Dựa trên cơ sở dữ liệu phổ ${ }^{1} \mathrm{H}$ NMR và MS, 4 đã được xác định là 1dotriacontanol.

Phổ của các hợp chất 5 và 6 phù hợp với của 2-(prop-1-ynyl)-5-(5,6-dihyroxyhexa-1,3diynyl)-thiophen và stigmasterol $3-O-\beta$-Dglucopyranosid, $\quad \beta$-sitosterol $\quad 3-O-\beta$-Dglucopyranosid đã được phân lập từ cành cây Cúc tần. Việc xác định cấu trúc các chất này từ cành cây Cúc tần đã được thông báo trong một bài báo trước [8].

\section{Kết luận}

Nghiên cứu đã đi sâu vào việc phân lập các hợp chất terpenoid và steroid từ cây Cúc tần $(P$. indica) ở Việt Nam và đã phân lập được 5 hợp chất và một hỗn hợp hai hợp chất sterol glucosid. 
Việc phân lập được taraxasterol, taraxasteryl acetat và stigmasterol ở lượng lớn có ý nghĩa khoa học và thực tiễn, do có các hoạt tính sinh học như chống viêm, chống ung thư và chống bệnh tiểu đường [9-11] các hợp chất này có thể có ứng dụng dược hoặc làm tiền chất tổng hợp các hợp chất có hoạt tính sinh học mới.

\section{Lời cảm ơn}

Nghiên cứu này được tài trợ bởi Quỹ phát triển Khoa học và Công nghệ quốc gia (NAFOSTED) trong đề tài mã số 104.012017.41.

\section{Tài liệu tham khảo}

[1] Đỗ Tất Lợi, Những cây thuốc và vị thuốc Việt Nam, NXB Hồng Đức, Thanh Hóa, 2014.

[2] H. Hussain, A. Al-Harrasi, G. Abba, N.U. Rheman, F. Mabood, I. Ahmed, M. Saleem, T. Van Ree, I.R. Green, S. Anwar, A. Badshah, A. Shah, I. Ali. The genus Pluchea: Phytochemistry, Traditional uses, and Biological activities. Chemistry \& Biodiversity 10 (2011) 1944-1969.

[3] Đoàn Thanh Tường, Phạm Hoàng Ngọc, Đỗ Đình Rãng. Nghiên cứu về một số cấu tử chính của tinh dầu Cúc tần. Thông báo khoa học Trường Đại học Sư phạm - Đại học Quốc gia Hà Nội 4 (199) (2015) 45-50.

[4] Vũ Việt Nam, Trần Ngọc Ninh, Mc Leod J., Nguyễn Xuân Dũng, Kết quả nghiên cứu một số cây thuộc họ Cúc, Tạp chí Dược liệu 4 (2) (1999) 44-47.

[5] Phan Minh Giang, Đỗ Thị Việt Hương, Nguyễn Ngọc Diệp, Nguyễn Mạnh Quyết, Một số hợp chất terpenoid và acid phenolic từ cây mẫn tưới, Tạp chí Dược liệu 23 (4) (2018) 195-199.

[6] L.M. Khalilov, A.Z. Khalilova, A.E.R. Shakurova, I.F. Nuriev, A.S. Kachala, A.S. Shashkov, U.M. Dzhemilev. PMR and ${ }^{13}$ C NMR spectra of biologically active compunds. XII. Taraxasterol and its acetate from the aerial part of Onopordum acanthium. Chemistry of Natural Compounds 39 (3) (2003) 285-288.

[7] L.J. Goad, T. Akihisha. Analysis of Sterols, Chapman \& Hall, London, 1997.

[8] Phan Minh Giang, Văn Thị Thanh Huyền, Đỗ Thị Việt Hương. Thành phần sterol, glycerol ester và thiophen trong cành cây cúc tần (Pluchea indica L.) của Việt Nam. Tạp chí Khoa học Đại học Quốc gia Hà Nội 34 (2) (2018) 78-82. https:// doi.org/10.25073/2588-1140/vnunst.4749.

[9] B. Sing, S.N. Ram, V.B. Pandey, V.K. Joshi, S. S. Gambhir. Studies on antiinflammatory activity of taraxasterol acetate from Echinops echinatus in rats and mice, Phytotherapy Research 5 (3) (1991) 103-106.

[10] Z. Krajcovicova, A. Vachálková, K. Horváthová. Taraxasterol and $\beta$-sitosterol: New naturally compounds with chemoprotective/ chemopreventive effects, Neoplasma 51 (2004) 407-414.

[11] J. Wang, M. Huang, J. Yang, X. Ma, S. Zheng, S. Deng, Y. Huang, X. Yang, P. Zhao. Antidiabetic activity of stigmasterol from soybean oil by targeting the GLUT4 glucose transporter. Food \& Nutrition Research 61 (1) (2017) 1364117. 